\title{
A Study on Determination of Turkish Adjective Pairs for Speech Sound
}

\author{
Ezgi Türk Gürkan¹ ${ }^{\bullet}$, Zerhan Yüksel Can² $\bullet$ \\ ${ }^{1} \mathrm{PhD}$ Student, Graduate School of Science and Engineering, Department of Architecture, Yıldız Technical University, Istanbul, \\ Turkey. (Principal contact for editorial correspondence), Email: ezgi.turkezgi@gmail.com \\ ${ }^{2}$ Prof. Dr., Faculty of Architecture, Department of Architecture, Ylldız Technical University, Istanbul, Turkey. Email: \\ zerhanyukselcan@gmail.com
}

\begin{abstract}
Purpose

Disagreement between the designer and the audience causes creation of acoustically uncomfortable spaces, eventually these disagreements cause financial loss. To describe a phenomenon with the same modifier is the key to settle the conflict. It is very important to reveal acoustic conditions and it is necessary to use a common terminology for this purpose. To this end subjective evaluation studies are frequently used, especially in terms of room acoustics. To define sound via adjective and adjective pairs is a method used in determining architectural acoustic conditions in halls, mostly used for music-function halls but subjective evaluation studies are not common for speech-function halls. Turkish adjectives for speech sound that determine audience preferences are missing. Adjectives obtained from translations of different languages do not show the same descriptive effect in a specific language. Therefore, it is important to identify adjectives that define sound for each language.

\section{Design/Methodology/Approach}

In order to determine the Turkish adjectives that define the speech sound, which are absent from the literature, a two-step survey was performed. Sets of modifiers associated with acoustics parameters -reverberation, clarity and loudness- were prepared, that include direct translations from common English modifiers as well as Turkish modifiers that were derived from expert opinion. These sets of adjectives were then presented to the subjects and they were asked to make choices among them.

\section{Findings}

The results of survey where modifiers defining speech sound are assessed are presented. The results show that some are unsuitable in Turkish despite the same word is used in subjective evaluations in English often. Based on the survey results, a questionnaire can be prepared for determining the user preferences in speech-function halls to increase the acoustic comfort conditions.
\end{abstract}

\section{Research Limitations/Implications}

Need of explaining the room acoustics parameters to the subjects.

Social/Practical Implications

It is thought that the questionnaire text will benefit the increase of the acoustic comfort conditions in the new and / or existing buildings and reduce economic loss.

\section{Originality/Value}

This study is the first research which examines Turkish adjectives to describe speech sound.

Keywords: Architectural acoustics, subjective acoustic evaluation, speech sound, speech-function halls, Turkish adjective pairs 


\section{INTRODUCTION}

To provide suitable acoustic conditions in accordance with the function of the space is indeed a major issue that has substantial social and economic consequences (Yılmaz Karaman \& Berber Üçkaya, 2015). Thus, careful design and constant improvements are needed to safeguard against acoustic problems. In the field of room acoustics, such improvements are typically made by taking into consideration during the design stage by listening on simulations or later on according to the comments of the in-situ audience. Preference surveys based on listening tests are the most used method for these studies. It is common to define the sound via adjective and / or adjective pairs in these surveys. Hence, the researchers' or designers' examination heavily depends on the ability of the audience to describe the sound as an adjective or an adjective pair in an articulate manner.

Despite being used often in defining sound of music, use of adjectives is not common in defining sound of speech. This may be due to the association of the sound of music with more subjective acoustic parameters than the sound of speech. Moreover, subjective evaluations of speech-function halls are mostly excluded from the scope of the studies in Turkey due to the absence of Turkish adjectives in particular. To match measurable or calculable values -objective parameters- and subjective evaluations has a primary importance in acoustics, as in any field of design. The objective parameters used in acoustic design is determined with international (British Standards Institution, 2009). A universal terminology unity is provided in this area by this standardization. On the other hand, the modifiers (adjectives or adjective pairs) to be used for matching objective parameters with subjective evaluations are unique to each language and are difficult to use effectively with direct translation from another language.

In this study, translated adjectives do not show the same descriptive effect to define sound and thus it is important to identify adjectives that define music and speech sound for one's native language is hypothesized.

Accordingly, a comprehensive literature research (some are shown in Table 2) is conducted on subjective evaluation of speech-function halls in Turkish which clearly showed a lack of such vocabulary and raised the need a study to determine the Turkish adjectives that can be used in subjective evaluations.

The aim of this study is to fill the observed gap and to help the researchers and designers to collect subjective evaluation on speechfunction halls. Towards this goal two-step survey experiment was performed to define Turkish modifiers. This methodology has been used in a collaborative study with Istanbul Technical University and Ylldız Technical University to choose words as annoyance modifiers in terms of noise in 1999 (Kurra, 2009), to be served by International Commission on Biological Effects of Noise as a recommendation to 
create a common terminology in terms of noise annoyance (Fields et al., 1998).

A two-step survey experiment is designed to determine Turkish adjectives as the first step and adjective pairs as the second step. Finally, based on the survey results, a list of modifiers is recommended for a questionnaire that can be used in subjective evaluation of speechfunction halls and help researchers and designers reduce complications that arise from lack of information.

\section{ROOM ACOUSTIC PARAMETERS ASSOCIATED WITH SPEECH SOUND AND THE TEST PROCEDURE}

Subjective studies of the acoustical characteristics of auditoria have shown that several quantities that can be obtained from measured impulse responses are correlated with particular subjective aspects of the acoustical character of an auditorium (British Standards Institution, 2009).

The relation between subjective aspect and objective quantities given in the standard can be seen in Table 1 .

Table 1. Adjectives used in the first step of the tests

\begin{tabular}{|l|l|}
\hline \multicolumn{1}{|c|}{ Subjective Listener Aspect (Subjective) } & \multicolumn{1}{c|}{ Acoustic Quantity (Objective) } \\
\hline Subjective level of sound & Sound strength, G (dB) \\
\hline Perceived reverberance & Early decay time, EDT (s) \\
\hline Perceived clarity of sound & Clarity, $\mathrm{C}_{80}(\mathrm{~dB})$ \\
& Definition, $\mathrm{D}_{50}$ \\
& Centre time, $\mathrm{T}_{\mathrm{s}}(\mathrm{ms})$ \\
\hline
\end{tabular}

In order to determine adjective pairs, room acoustics parameters that are directly related to speech function were first determined. These parameters and their definitions are listed below:

- $\quad$ Reverberation ("Yansışım, Çınlama" in Turkish): After the sound source has been silenced, a certain amount of time is required to absorb the existing sound energy so that it cannot be heard (Furrer et al., 1964). This phenomenon that occurs over time is called reverberation.

Reverberation time is the duration required for the space-averaged sound energy density in an enclosure to decrease by $60 \mathrm{~dB}$ after the source emission has stopped. The reverberation time of a room was once regarded as the predominant indicator of its acoustical properties. While reverberation time continues to be regarded as a significant parameter, there is reasonable agreement that other types of measurements, such as relative sound pressure levels, early/late energy ratios, lateral energy fractions, interaural cross-correlation functions and background noise levels, are needed for a more complete evaluation of the acoustical quality of rooms (British Standards Institution, 2009). It is clear that the reverberation has a significant effect on the acoustic behaviour of the hall. It is a phenomenon that plays an important role in all areas of room acoustics and is least discussed parameter in evaluating the acoustic quality of any type of space (Kuttruff, 1979). 
- $\quad$ Clarity ("Netlik" in Turkish): Clarity is an early-to-late arriving sound energy ratio. This can be calculated for either a $50 \mathrm{~ms}$ or an $80 \mathrm{~ms}$ early time limit, depending on whether the results are intended to relate to conditions for speech or music, respectively. The early time limit of either $50 \mathrm{~ms}$ or $80 \mathrm{~ms}$ is defined as clarity (British Standards Institution, 2009).

Clarity describes the degree to which every detail of the performance is perceived. This parameter is to a large extent a property complementary to reverberation. The more the early sound dominates, the higher the impression of clarity (Gade, 2007). Clarity is defined as the ratio of early to late arriving sound, with the distinction for speech made at $50 \mathrm{~ms}$ between early and late (Harvie-Clark et al., 2014).

- Level of Sound, Loudness ("Düzey, Gürlük" in Turkish): The sound strength is the logarithmic ratio of the sound energy of the measured impulse response to that of the response measured in a free field at a distance of $10 \mathrm{~m}$ from the sound source (British Standards Institution, 2009). To put it simply, it can be described as the level of sound heard in the receiver point (Barron, 2009). It is clear that the sound must be loud enough at all receiver (listener) locations for all audiences to hear well. The voice can be raised naturally to adjust the loudness according to the size of the audience speaker is addressing; however, this has a certain limit.

Next, a literature research is conducted to compile a list of adjectives that are used in the description of the parameters above -reverberation, clarity and loudness- and adjectives related to general evaluation of sound. Hence, the evaluations are disintegrated into four categories, which are;

- $\quad$ Reverberation,
- $\quad$ Loudity,
- $\quad$ General Evaluation.

The test procedure is consisted of two steps;

- Selection of the adjectives,

- $\quad$ Selection of the adjective pairs.

In order to conduct the test procedure successfully, a training session is organized with the subjects where they listened to audio recordings representing the extreme states of reference acoustic parameters to get familiar with how these parameters sound. Audio recordings used in the listening session are obtained from the simulation of a hall designed as a reference. Odeon v.15 is used for the simulations. The simulation variations of the hall with high and low values of the room acoustics parameters (extreme states of reverberation time, clarity and loudness) are modelled. Speech signals recorded under anechoic conditions are added to the prepared hall simulations and auralisations are obtained for the extreme conditions. Anechoic recordings are obtained from Odeon Room Acoustics Software's database (Odeon A / S, 2019). 
Twenty subjects, five of which are trained in acoustics, participated in the two-step survey where they were asked to select among the adjectives and pairs of adjectives that are given in the lists. Selections made by two types of subjects are weighted differently: weight of 2 is issued for the subjects trained in acoustics. Thus, the text data is obtained by integrating 25 total points to 100 .

The tests conducted are independent of any hall and aimed only to identify the sound. It is aimed to identify the appropriate Turkish modifiers that are usually being used in defining an acoustic environment, to correlate relationship between the listener's subjective aspect objective quantities. Therefore, any listening was not included during the tests.

\section{FIRST STEP OF THE TESTS, DETERMINATION OF ADJECTIVES}

In the first step of the tests, subjects are given adjectives that define the room acoustic parameters and general evaluation, and they are asked to select the adjectives which they consider appropriate to define the relevant category. The descriptive adjective list is expanded by adding the adjectives obtained in the researches. Adjectives used in the first step of the tests are given in Table 2 .

Table 2. Adjectives used in the first step of the tests

\begin{tabular}{|c|c|c|c|}
\hline Reverberation & Clarity & Loudness & General Evaluation \\
\hline $\begin{array}{l}\text { Dead (Ölü) } \\
\text { (Vural, 2009) }\end{array}$ & $\begin{array}{l}\text { Complicated } \\
\text { (Karışık) } \\
\text { (Vural, 2009) } \\
\text { (Özçevik, 2012) }\end{array}$ & $\begin{array}{l}\text { Quiet (Sessiz) } \\
\text { (Vural, 2009) } \\
\text { (Özçevik, 2012) }\end{array}$ & $\begin{array}{l}\text { I Found it Hard } \\
\text { (Zorlandim) } \\
\text { (Orhan, 2019) }\end{array}$ \\
\hline $\begin{array}{l}\text { Dry (Kuru) } \\
\text { (Farina, 2001) }\end{array}$ & $\begin{array}{l}\text { Clear (Açlk) } \\
\text { (Vural, 2009) }\end{array}$ & $\begin{array}{l}\text { Loud (Yüksek Sesli) } \\
\text { (Vural, 2009) } \\
\text { (Orhan, 2019) }\end{array}$ & $\begin{array}{l}\text { I Didn't Find it Hard } \\
\text { (Zorlanmadım) }\end{array}$ \\
\hline $\begin{array}{l}\text { Live (Canlı) } \\
\text { (Vural, 2009) } \\
\text { (Çoktaș, 2019) }\end{array}$ & $\begin{array}{l}\text { Blurred } \\
\text { (Bulanık) } \\
\text { (Savcl Özgüven, } \\
2015)\end{array}$ & $\begin{array}{l}\text { Weak (Zayıf) } \\
\text { (Farina, 2001) } \\
\text { (Özçevik, 2012) }\end{array}$ & $\begin{array}{l}\text { I Made Effort to } \\
\text { Understand } \\
\text { (Anlamak için Çaba } \\
\text { Gösterdim) }\end{array}$ \\
\hline $\begin{array}{l}\text { Reverberant } \\
\text { (Çınlamalı) } \\
\text { (Farina, 2001) }\end{array}$ & $\begin{array}{l}\text { Neat (Net) } \\
\text { (Özçevik, 2012) } \\
\text { (Berber Üçkaya, } \\
2014) \\
\text { (Savcl Özgüven, } \\
2015) \\
\text { (Çoktaş, 2019) }\end{array}$ & $\begin{array}{l}\text { Strong (Güçlü) } \\
\text { (Farina, 2001) } \\
\text { (Özçevik, 2012) } \\
\text { (Çoktaş, 2019) }\end{array}$ & $\begin{array}{l}\text { I Didn't Make Effort } \\
\text { to Understand } \\
\text { (Anlamak için Çaba } \\
\text { Göstermedim) }\end{array}$ \\
\hline Occupied (Dolu) & $\begin{array}{l}\text { Murmuring } \\
\text { (Miriltılı) }\end{array}$ & Close (Yakın) & $\begin{array}{l}\text { I am Satisfied } \\
\text { (Memnun Kaldım) } \\
\text { (Farina, 2001) }\end{array}$ \\
\hline Unoccupied (Boş) & $\begin{array}{l}\text { Understandable } \\
\text { (Anlaşılır) }\end{array}$ & $\begin{array}{l}\text { Far (Uzak) } \\
\text { (Vural, 2009) }\end{array}$ & $\begin{array}{l}\text { I am Disturbed } \\
\text { (Rahatsız Oldum) } \\
\text { (Farina, 2001) }\end{array}$ \\
\hline $\begin{array}{l}\text { Non-Echoed } \\
\text { (Yankı Yok) }\end{array}$ & $\begin{array}{l}\text { Easy to } \\
\text { Understand } \\
\text { (Kolay Anlașılır) }\end{array}$ & $\begin{array}{lr}\text { Speaker } & \text { Should } \\
\text { Make Effort } & \\
\text { (Konuşmacl } & \text { Çaba } \\
\text { Göstermeli) } & \end{array}$ & - \\
\hline
\end{tabular}




\begin{tabular}{|c|c|c|c|}
\hline $\begin{array}{l}\text { Echoed } \\
\text { Var) } \\
\text { (Berber } \\
2014) \\
\text { (Savcl } \\
2015)\end{array}$ & $\begin{array}{l}\text { Non- } \\
\text { Understandable } \\
\text { (Anlaşılmaz) }\end{array}$ & $\begin{array}{l}\text { Speaker Doesn't } \\
\text { Need to Make Effort } \\
\text { (Konuşmacının Çaba } \\
\text { Göstermesine Gerek } \\
\text { Yok) }\end{array}$ & - \\
\hline $\begin{array}{l}\text { Large (Büyük) } \\
\text { (Berber Üçkaya, } \\
\text { 2014) }\end{array}$ & $\begin{array}{l}\text { Fast (Hızlı) } \\
\text { (Özçevik, 2012) }\end{array}$ & $\begin{array}{l}\text { Sound Level is not } \\
\text { Enough } \\
\text { (Ses Düzeyi Yetersiz) }\end{array}$ & - \\
\hline Small (Küçük) & $\begin{array}{l}\text { Slow (Yavaş) } \\
\text { (Özçevik, 2012) }\end{array}$ & $\begin{array}{l}\text { Sound Level is } \\
\text { Enough } \\
\text { (Ses Düzeyi Yeterli) }\end{array}$ & - \\
\hline - & $\begin{array}{l}\text { At Normal Speed } \\
\text { (Normal Hizda) }\end{array}$ & - & - \\
\hline
\end{tabular}

The selections made in the test are given in Table 3.

Table 3. Selections made in the first step of the tests

\begin{tabular}{|c|c|c|c|c|c|c|c|c|c|c|c|c|c|c|c|c|c|c|c|}
\hline Adjective & \multicolumn{19}{|c|}{ Subject } \\
\hline $\begin{array}{l}\text { Dead } \\
\text { (Ölü) }\end{array}$ & & o & 3 & 3 & & & 3 & & & & 3 & 3 & & 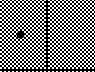 & & & & & \\
\hline $\begin{array}{l}\text { Dry } \\
\text { (Kuru) }\end{array}$ & : & & & & & & 3 & e. & & & & & & & & & & & \\
\hline $\begin{array}{l}\text { Live } \\
\text { (Canlı) }\end{array}$ & : & 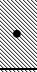 & 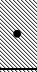 & 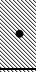 & $s$ & 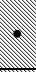 & 3 & : & & 3 & o & - & o & $\cdot 0$ & & ? & & & 3 \\
\hline $\begin{array}{l}\text { Reverberant } \\
\text { (Çınlamalı) }\end{array}$ & : & & $s$ & sis & & 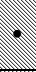 & 3 & : & & & & - & & : & . & 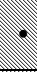 & o & ? & 3 \\
\hline $\begin{array}{l}\text { Occupied } \\
\text { (Dolu) }\end{array}$ & : & & & 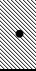 & & & & is: & 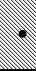 & 3 & & ( & & & & & & 3 & 3 \\
\hline $\begin{array}{l}\text { Unoccupied } \\
\text { (Boș) }\end{array}$ & : & & & 3 & & & & : & 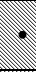 & & $\sqrt{3}$ & - & & & & & & & 8 \\
\hline $\begin{array}{l}\text { Non-Echoed } \\
\text { (Yankı Yok) }\end{array}$ & : & 3 & 3 & 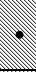 & - & & 3 & * & ? & 3 & & - & & +0 & . & $\sqrt{3}$ & 3 & ? & s \\
\hline $\begin{array}{l}\text { Echoed } \\
\text { (Yankı Var) }\end{array}$ & : & 3 & 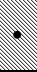 & 3 & ? & e & 3 & *. & $s$ & & o & (. & o & 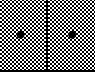 & *. & 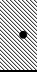 & ? & e & 3 \\
\hline $\begin{array}{l}\text { Large } \\
\text { (Büyük) }\end{array}$ & : & & & & & & & . & $s$ & & & & & 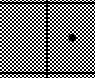 & & & & o & 3 \\
\hline $\begin{array}{l}\text { Small } \\
\text { (Küçük) }\end{array}$ & : & & & & & & & . & 3 & & & & & : & & & & ? & 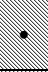 \\
\hline $\begin{array}{l}\text { Complicated } \\
\text { (Karışı) }\end{array}$ & & & o & & & & & & & & & (3) & & & : & & & & \\
\hline $\begin{array}{l}\text { Clear } \\
\text { (Açık) }\end{array}$ & & & o & ? & & ? & & * & & o & & ? & & : & & & & & \\
\hline $\begin{array}{l}\text { Blurred } \\
\text { (Bulanık) }\end{array}$ & 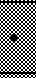 & & & ? & & & - & * & & & & - & & +0 & & & o & & \\
\hline $\begin{array}{l}\text { Neat } \\
\text { (Net) }\end{array}$ & : & ? & - & ? & - & - & 3 & * & 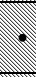 & 3 & - & * & & 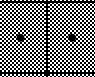 & 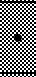 & 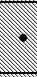 & o & o & 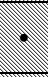 \\
\hline $\begin{array}{l}\text { Murmuring } \\
\text { (Miriltıll) }\end{array}$ & & & ? & - & & & & * & & & & e. & 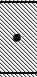 & : & : & & ? & & \\
\hline $\begin{array}{l}\text { Understandable } \\
\text { (Anlașilır) }\end{array}$ & : & e. & ? & 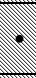 & o & - & ? & * & . & 3 & - & (.) & & 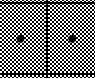 & $*$ & ? & o & 3 & so \\
\hline $\begin{array}{l}\text { Esay to } \\
\text { Understand } \\
\text { (Kolay Anlașlır) }\end{array}$ & : & & & * & ? & & 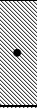 & *: & & 3 & 0 & 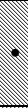 & & : & : & 3 & e & 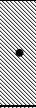 & 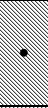 \\
\hline $\begin{array}{l}\text { Non- } \\
\text { Understandable } \\
\text { (Anlașılmaz) }\end{array}$ & : & ? & 3 & 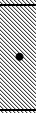 & (- & & o & * & o & & 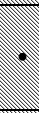 & (.) & & $\cdot 0$ & *: & 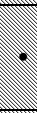 & 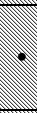 & 3 & 3 \\
\hline $\begin{array}{l}\text { Fast } \\
\text { (Hızlı) }\end{array}$ & & & (- & & & & & $*$ & c. & & (3) & 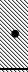 & & 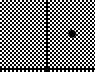 & & sis & & o & \\
\hline $\begin{array}{l}\text { Slow } \\
\text { (Yavaş) }\end{array}$ & & & e & & & & & . & 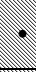 & & $\sqrt{3}$ & e & & 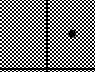 & & 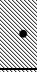 & & o & \\
\hline $\begin{array}{ll}\text { At } & \text { Normal } \\
\text { Speed } & \\
\end{array}$ & & & o & & & ? & & * & & o & 3 & ? & & : & & o & & ( & \\
\hline
\end{tabular}




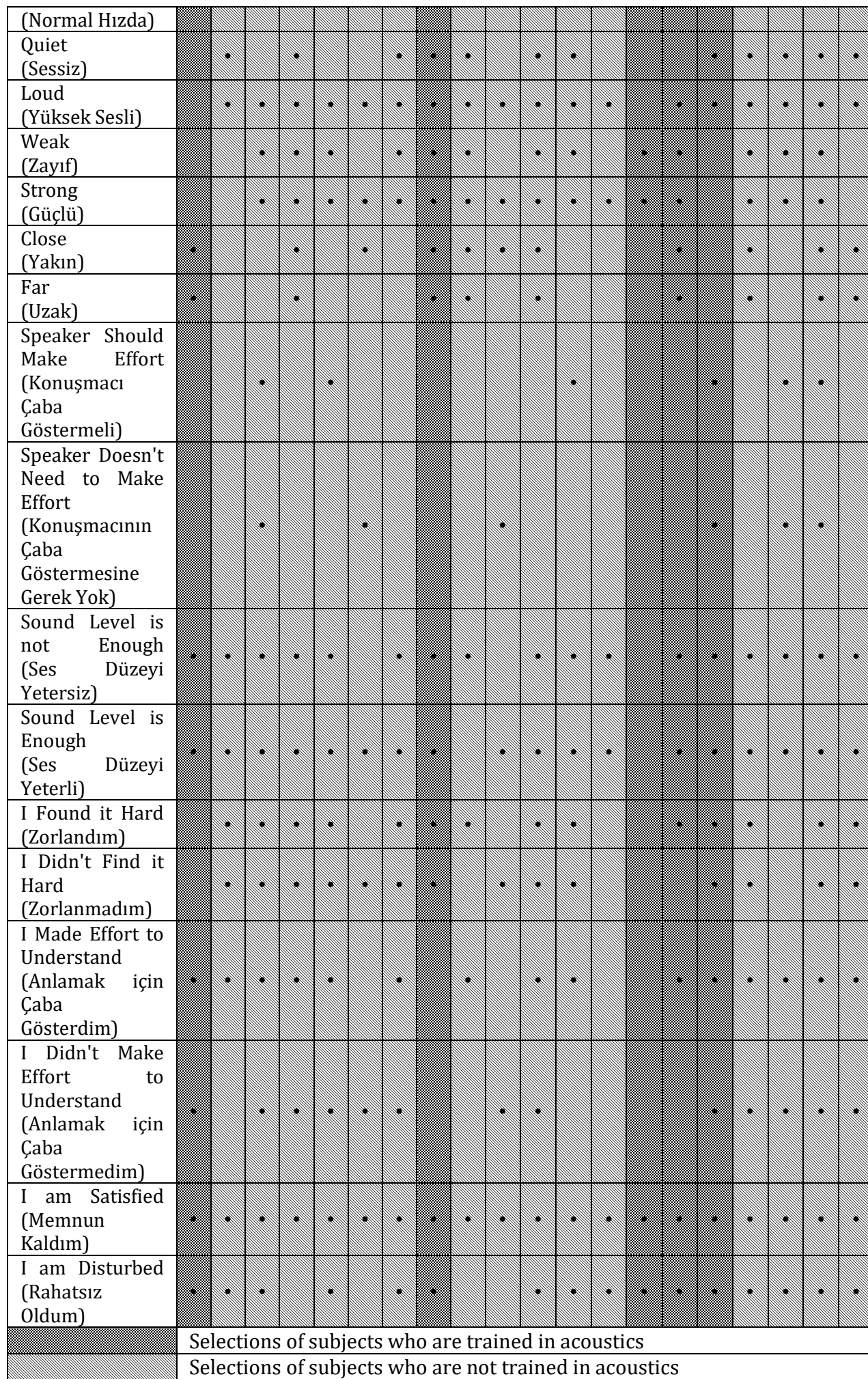

Table 4 shows the ratio of the selections decreasingly made by the subjects for each of the four categories.

Table 4. Data obtained in the first step of the tests

\begin{tabular}{|c|c|c|c|}
\hline Category & Scale & $\begin{array}{c}\text { Adjective } \\
\end{array}$ & Preferance Ratio (\%) \\
\hline \multirow{3}{*}{ 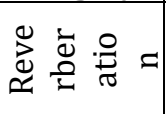 } & \multirow{3}{*}{ Low } & Non-Echoed (Yankı Yok) & 88 \\
\hline & & Occupied (Dolu) & 40 \\
\hline & & Small (Küçük) & 36 \\
\hline
\end{tabular}




\begin{tabular}{|c|c|c|c|}
\hline & & Dead (Ölü) & 32 \\
\hline & & Dry (Kuru) & 20 \\
\hline & \multirow{5}{*}{ High } & Echoed (Yankı Var) & 96 \\
\hline & & Live (Canlı) & 80 \\
\hline & & Reverberant (Çınlamalı) & 68 \\
\hline & & Large (Büyük) & 36 \\
\hline & & Unoccupied (Boș) & 36 \\
\hline \multirow{11}{*}{ 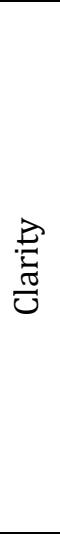 } & \multirow{5}{*}{ Low } & $\begin{array}{l}\text { Non-Understandable } \\
\text { (Anlașılmaz) }\end{array}$ & 88 \\
\hline & & Blurred (Bulanık) & 48 \\
\hline & & Murmuring (Mırıltılı) & 44 \\
\hline & & Fast (Hızlı) & 40 \\
\hline & & Complicated (Karıșık) & 16 \\
\hline & & Understandable (Anlaşılır) & 96 \\
\hline & & Neat (Net) & 96 \\
\hline & & $\begin{array}{l}\text { Easy to Understand (Kolay } \\
\text { Anlaşılır) }\end{array}$ & 72 \\
\hline & High & $\begin{array}{lll}\text { At Normal Speed } & \text { (Normal } \\
\text { Hizda) } & & \\
\end{array}$ & 44 \\
\hline & & Slow (Yavaș) & 40 \\
\hline & & Clear (Açlk) & 36 \\
\hline & & $\begin{array}{l}\text { Sound Level is not Enough (Ses } \\
\text { Düzeyi Yetersiz) }\end{array}$ & 84 \\
\hline & & Weak (Zayıf) & 64 \\
\hline & Low & Quiet (Sessiz) & 56 \\
\hline & & Far (Uzak) & 48 \\
\hline $\mathscr{d}$ & & $\begin{array}{l}\text { Speaker Should Make Effort } \\
\text { (Konușmacl Çaba Göstermeli) }\end{array}$ & 28 \\
\hline$\frac{\tilde{E}}{\tilde{J}}$ & & $\begin{array}{l}\text { Sound Level is Enough (Ses } \\
\text { Düzeyi Yeterli) }\end{array}$ & 88 \\
\hline & & Loud (Yüksek Sesli) & 84 \\
\hline & High & Strong (Güçlü) & 76 \\
\hline & Hign & Close (Yakın) & 56 \\
\hline & & $\begin{array}{l}\text { Speaker Doesn't Need to Make } \\
\text { Effort (Konuşmacının Çaba } \\
\text { Göstermesine Gerek Yok) }\end{array}$ & 28 \\
\hline & & $\begin{array}{l}\text { I am Disturbed } \text { (Rahatsız } \\
\text { Oldum) }\end{array}$ & 84 \\
\hline$\stackrel{\overbrace{}}{\stackrel{0}{\pi}}$ & Negative & $\begin{array}{l}\text { I Made Effort to Understand } \\
\text { (Anlamak için Çaba Gösterdim) }\end{array}$ & 72 \\
\hline$\stackrel{\pi}{\Xi}$ & & I Found it Hard (Zorlandım) & 68 \\
\hline 蛋 & & 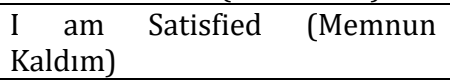 & 100 \\
\hline$\stackrel{\frac{\pi}{2}}{\frac{\pi}{0}}$ & Positive & $\begin{array}{l}\text { Didn't Find it } \text { Hard } \\
\text { (Zorlanmadım) }\end{array}$ & 64 \\
\hline$\circlearrowleft$ & & $\begin{array}{l}\text { I Didn't Make Effort to } \\
\text { Understand Anlamak için Çaba } \\
\text { Göstermedim) }\end{array}$ & 60 \\
\hline
\end{tabular}

During the tests, various comments are obtained from the subjects. These comments suggest that there are different adjectives that may be more descriptive for the selected room acoustic parameters for tests. On the other hand, some adjectives are found to be difficult to understand by the subjects. In the second step of the tests the comments of the subjects are taken into consideration for determining the adjective pairs to be used.

SECOND STEP OF THE TESTS, DETERMINATION OF ADJECTIVE PAIRS 
The second step tests are performed with the subjects of the previous step. Before the tests, the room acoustics parameters that are desired to be defined are explained briefly to the subjects and the audio samples are played to help the subjects to identify them.

In the second step of the tests, subjects are asked to select adjective pairs that they consider appropriate to be used for describing speech sound. For the sake of clarity maximum five adjective pairs for each category is considered to be sufficient in the questionnaire for determining audience preferences in speech-function halls per the aim of the study. Therefore, subjects are expected to limit their selections with five adjective pairs per category they consider appropriate.

The adjective pairs used in the second step of the tests are given in Table 5.

Table 5. Adjective pairs used in the second step of the tests

\begin{tabular}{|c|c|}
\hline Reverberation & Clarity \\
\hline $\begin{array}{l}\text { Dead - Live } \\
\text { (Ölü - Canlı) }\end{array}$ & $\begin{array}{l}\text { Complicated - Clear } \\
\text { (Karıșık - Açı) }\end{array}$ \\
\hline $\begin{array}{l}\text { Dry - Live } \\
\text { (Kuru - Canlı) }\end{array}$ & $\begin{array}{l}\text { Blurred - Neat } \\
\text { (Bulanık - Net) }\end{array}$ \\
\hline $\begin{array}{l}\text { Dry - Reverberant } \\
\text { (Kuru - Çınlamalı) }\end{array}$ & $\begin{array}{l}\text { Murmuring - Understandable } \\
\text { (Mırıltıll - Anlașılır) }\end{array}$ \\
\hline $\begin{array}{l}\text { Dead - Reverberant } \\
\text { (Ölü - Çınlamalı) }\end{array}$ & $\begin{array}{l}\text { Non-Understandable - Understandable } \\
\text { (Anlașllmaz - Anlașılır) }\end{array}$ \\
\hline $\begin{array}{l}\text { Non-Reverberant - Reverberant } \\
\text { (Çınlamasız - Çınlamalı) }\end{array}$ & $\begin{array}{l}\text { Non-Understandable - Easy to } \\
\text { Understand } \\
\text { (Anlaşılmaz - Kolay Anlaşılır) }\end{array}$ \\
\hline $\begin{array}{l}\text { Howling- Non-Howling } \\
\text { Uğultulu - Uğultusuz }\end{array}$ & $\begin{array}{l}\text { Hard to Understand - Easy to Understand } \\
\text { (Anlașılması Zor - Kolay Anlașilır) }\end{array}$ \\
\hline $\begin{array}{l}\text { Echoed - Non-Echoed } \\
\text { (Yankı Var (Yankılı) - Yankı Yok } \\
\text { (Yankısız)) }\end{array}$ & 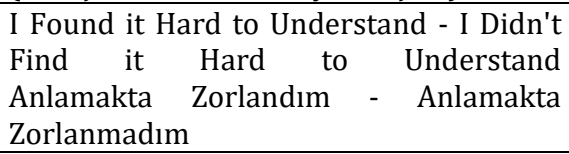 \\
\hline $\begin{array}{l}\text { Large Hall - Small Hall } \\
\text { (Büyük Salon - Küçük Salon) }\end{array}$ & $\begin{array}{l}\text { Speaker Should Talk OnebyOne - Speaker } \\
\text { is Talking One } \quad \text { by } \\
\text { (Tane Tane Konuşmalı - Tane tane } \\
\text { Konușuyor) }\end{array}$ \\
\hline $\begin{array}{l}\text { Occupied - Unoccupied } \\
\text { (Dolu - Boș) }\end{array}$ & $\begin{array}{l}\text { Not One by One - One by One } \\
\text { (Tane Tane Değil - Tane Tane) }\end{array}$ \\
\hline $\begin{array}{l}\text { Absorbent - Non-Absorbent } \\
\text { (Yutucu - Yutucu Değil) }\end{array}$ & $\begin{array}{l}\text { Fast - At Normal Speed } \\
\text { (Hızlı - Normal Hızda) }\end{array}$ \\
\hline $\begin{array}{l}\text { Absorbent - Reverberant } \\
\text { (Yutucu - Çınlamalı) }\end{array}$ & $\begin{array}{l}\text { Fast - Slow } \\
\text { (Hızlı - Yavaş) }\end{array}$ \\
\hline Loudness & General Evaluation \\
\hline $\begin{array}{l}\text { Quiet - Loud } \\
\text { (Sessiz - Yüksek Sesli) }\end{array}$ & $\begin{array}{l}\text { I Found it Hard to Listen - I Didn't Find it } \\
\text { Hard to Listen } \\
\text { (Dinlerken Zorlandım - Dinlerken } \\
\text { Zorlanmadım) }\end{array}$ \\
\hline $\begin{array}{l}\text { Weak Sound - Strong Sound } \\
\text { (Zayıf Ses - Güçlü Ses) }\end{array}$ & $\begin{array}{l}\text { I Found it Hard - I Didn't Find it Hard } \\
\text { (Zorlandım - Zorlanmadım) }\end{array}$ \\
\hline $\begin{array}{l}\text { Speaker is Close - Speaker is Far Away } \\
\text { (Konuşmacı Yakın Konumda - Konuşmacı } \\
\text { Uzak Konumda) }\end{array}$ & $\begin{array}{lcc}\text { Acoustical Environment is Bad - } \\
\text { Acoustical } & \text { Environment is Good } \\
\text { (Akustik Ortam Kötü - Akustik Ortam İyi) }\end{array}$ \\
\hline $\begin{array}{l}\text { Low Sound-Loud Sound } \\
\text { (Alçak Ses - Yüksek Ses) }\end{array}$ & $\begin{array}{l}\text { I am Pleased- I m Uncomfortable } \\
\text { (Memnunum - Rahatsızım) }\end{array}$ \\
\hline $\begin{array}{l}\text { I Found it Hard to Hear- I Didn't Find it } \\
\text { Hard to Hear } \\
\text { (Duymakta Zorlandım } \quad-\quad \text { Duymakta }\end{array}$ & $\begin{array}{l}\text { I am Satisfied - I am Disturbed } \\
\text { (Memnun Oldum - Rahatsız Oldum) }\end{array}$ \\
\hline
\end{tabular}




\begin{tabular}{|l|l|}
\hline Zorlanmadım) & \\
\hline $\begin{array}{l}\text { Careless-Related } \\
\text { (Umursamaz - İlgili) }\end{array}$ & I Prefer the Hall - I Don't Prefer the Hall \\
& (Salonu Tercih Ederim - Salonu Tercih \\
\hline & Etmem) \\
\hline & I Made Effort While Listening - I Didn't \\
& Make Effort While Listening \\
& (Dinlerken Craba Gösterdim - Dinlerken \\
& Çaba Göstermedim) \\
\hline
\end{tabular}

The selections made in the second step tests are given in Table 6.

Table 6. Selections made in the second step of the tests

\begin{tabular}{|c|c|c|c|c|c|c|c|c|c|c|c|c|c|c|c|c|c|c|c|c|}
\hline Adjective Pair & & & & & & & & & & Sul & jec & & & & & & & & & \\
\hline $\begin{array}{l}\text { Dead - } \quad \text { Live } \\
\text { (Ölü - Canlı) }\end{array}$ & * & & & & & & & . & & 3 & v & 3 & 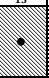 & & : & & 3 & & 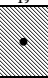 & 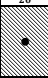 \\
\hline $\begin{array}{l}\text { Dry - Live } \\
\text { (Kuru - Canlı) }\end{array}$ & & & & & & & & & & & & & & 8 & & & & & & \\
\hline $\begin{array}{l}\text { Dry } \\
\text { Reverberant } \\
\text { (Kuru } \\
\text { Çוnlamalı) }\end{array}$ & & 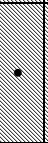 & & & & & - & & & & & & & . & & & & & & \\
\hline $\begin{array}{l}\text { Dead } \\
\text { Reverberant } \\
\text { (Ölü - Çınlamalı) }\end{array}$ & & & & & & & & & & & & & & & & & & & & \\
\hline $\begin{array}{l}\text { Non- } \\
\text { Reverberant } \\
\text { Reverberant } \\
\text { (Çınlamasız } \\
\text { Çınlamalı) }\end{array}$ & 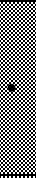 & & 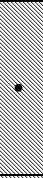 & 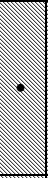 &. & 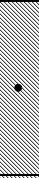 & 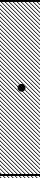 & : & 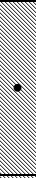 & 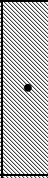 & 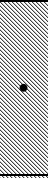 & 3 & & * & * & & 3 & 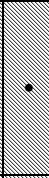 & 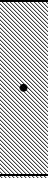 & 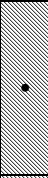 \\
\hline $\begin{array}{l}\text { Howling - Non- } \\
\text { Howling } \\
\text { (Uğultulu } \\
\text { Uğultusuz) }\end{array}$ & & 3 & & & & & & & 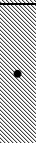 & & . & & ? & & 8 & 8 & . & & 3 & \\
\hline $\begin{array}{l}\text { Echoed - } \\
\text { Ecnoed } \\
\text { (Yankl } \\
\text { (Yankılı) - Yankı } \\
\text { Yok (Yankısı)) }\end{array}$ & & 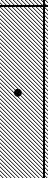 & 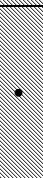 & 3 & 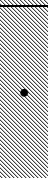 & $y$ & 3 & : & 3 & 3 & $y$ & & 3 & 0 & . & - & & $\sqrt{3}$ & & . \\
\hline $\begin{array}{l}\text { Large Hall - } \\
\text { Small Hall } \\
\text { (Büyük Salon - } \\
\text { Küçük Salon) }\end{array}$ & & 3 & ? & 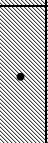 & 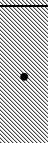 & 3 & 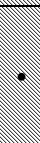 & & e & & & & & & & - & & & & \\
\hline $\begin{array}{ll}\text { Occupied } & - \\
\text { Unoccupied } & \\
\text { (Dolu-Boş) }\end{array}$ & & & 3 & 3 & 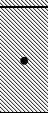 & & & ? & & & & & $\sqrt{3}$ & & & & & & 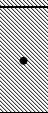 & \\
\hline $\begin{array}{l}\text { Absorbent } \\
\text { Non-Absorbent } \\
\text { (Yutucu } \\
\text { Yutucu Değil) }\end{array}$ & . & & 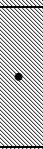 & $\sqrt{3}$ & & 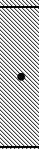 & & & & & & & (2) & & & & 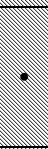 & & & \\
\hline $\begin{array}{ll}\text { Absorbent } & - \\
\text { Reverberant } & \\
\text { (Yutucu } & \text { - } \\
\text { Çınlamalı) } & \end{array}$ & & & & & & 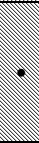 & . & . & & & & 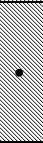 & & . & : & 0 & 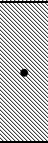 & & & 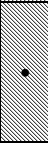 \\
\hline $\begin{array}{l}\text { Complicated - } \\
\text { Clear } \\
\text { (Karıșlk - Açık) }\end{array}$ & & & & $\sqrt{3}$ & & $\sqrt{3}$ & 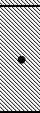 & & & $\sqrt{3}$ & 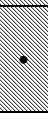 & & & & & & & & & \\
\hline $\begin{array}{l}\text { Blurred - Neat } \\
\text { (Bulanık - Net) }\end{array}$ & : & & & 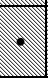 & 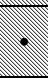 & 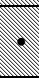 & 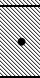 & . & & & & 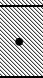 & 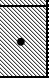 & : & 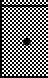 & 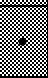 & 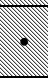 & 3 & 3 & \\
\hline $\begin{array}{l}\text { Murmuring - } \\
\text { Understandable } \\
\text { (Mırıltılı } \\
\text { Anlaşılır) } \\
\end{array}$ & & 3 & 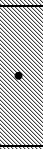 & & . & & & & 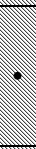 & 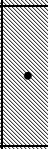 & & & & & & . & & 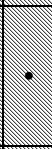 & & \\
\hline $\begin{array}{l}\text { Non- } \\
\text { Understandable } \\
- \\
\text { Understandable }\end{array}$ & 8 & 0 & 3 & 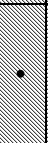 & & 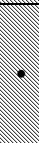 & 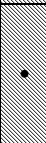 & & 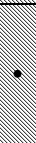 & & & 3 & & 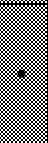 & ? & & . & 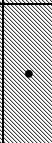 & 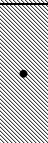 & . \\
\hline
\end{tabular}




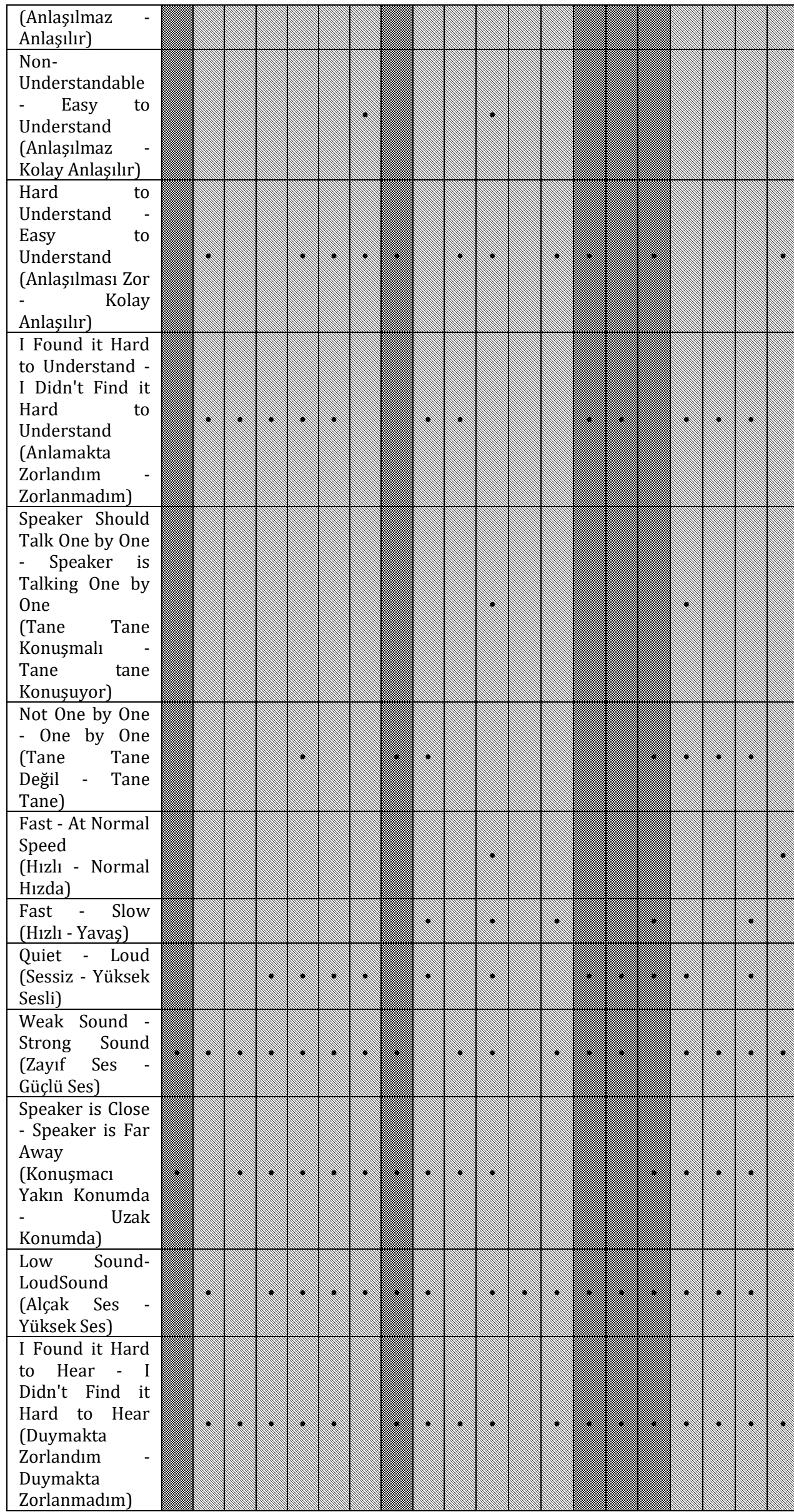




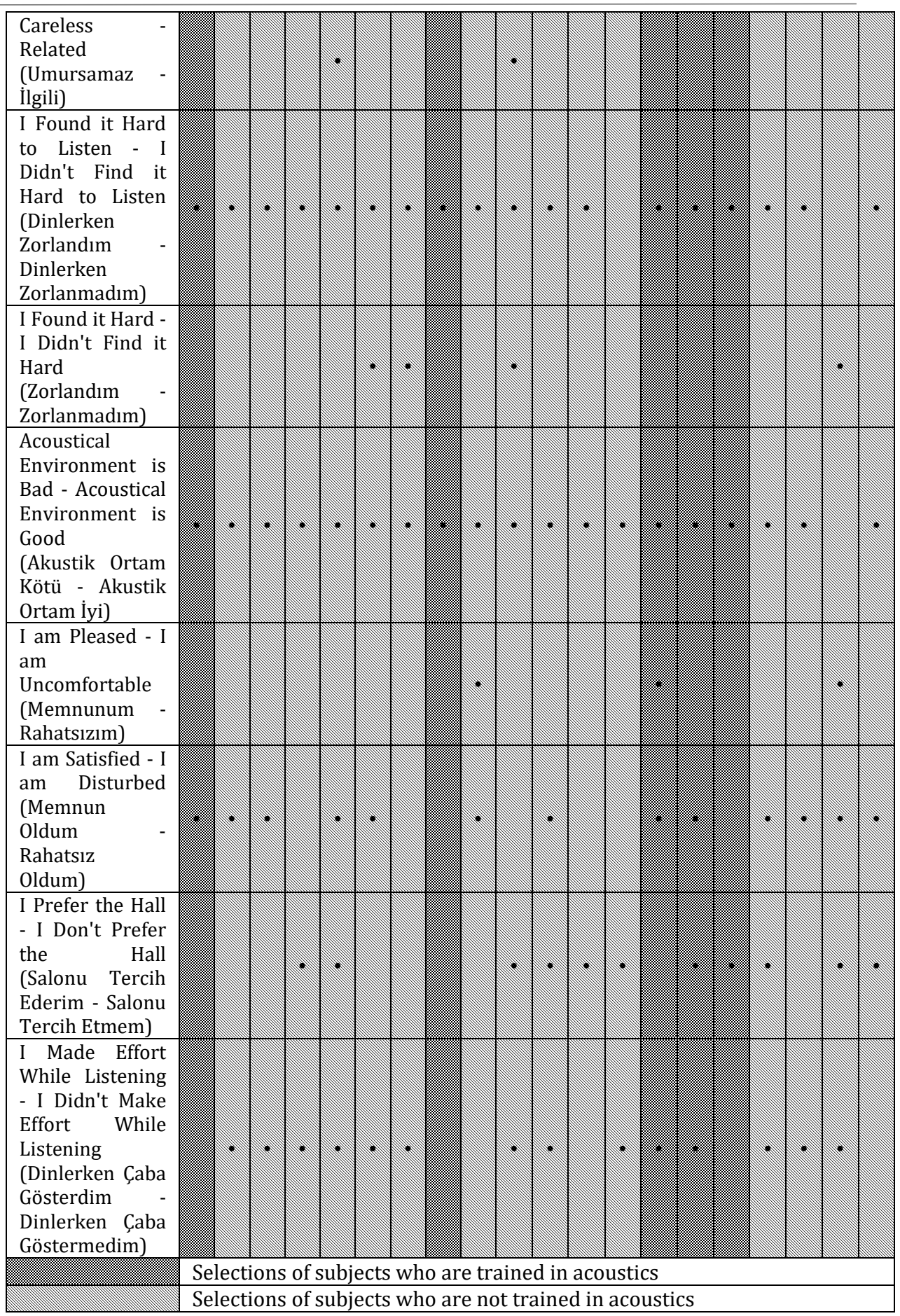

The selections made for adjective pairs belonging to different categories can be seen in Figures 1-4. 
Figure 1. Adjective Pair Selections for Reverberation Category

Figure 2. Adjective Pair Selections for Clarity Category

Figure 3. Adjective Pair Selections for Loudness Category
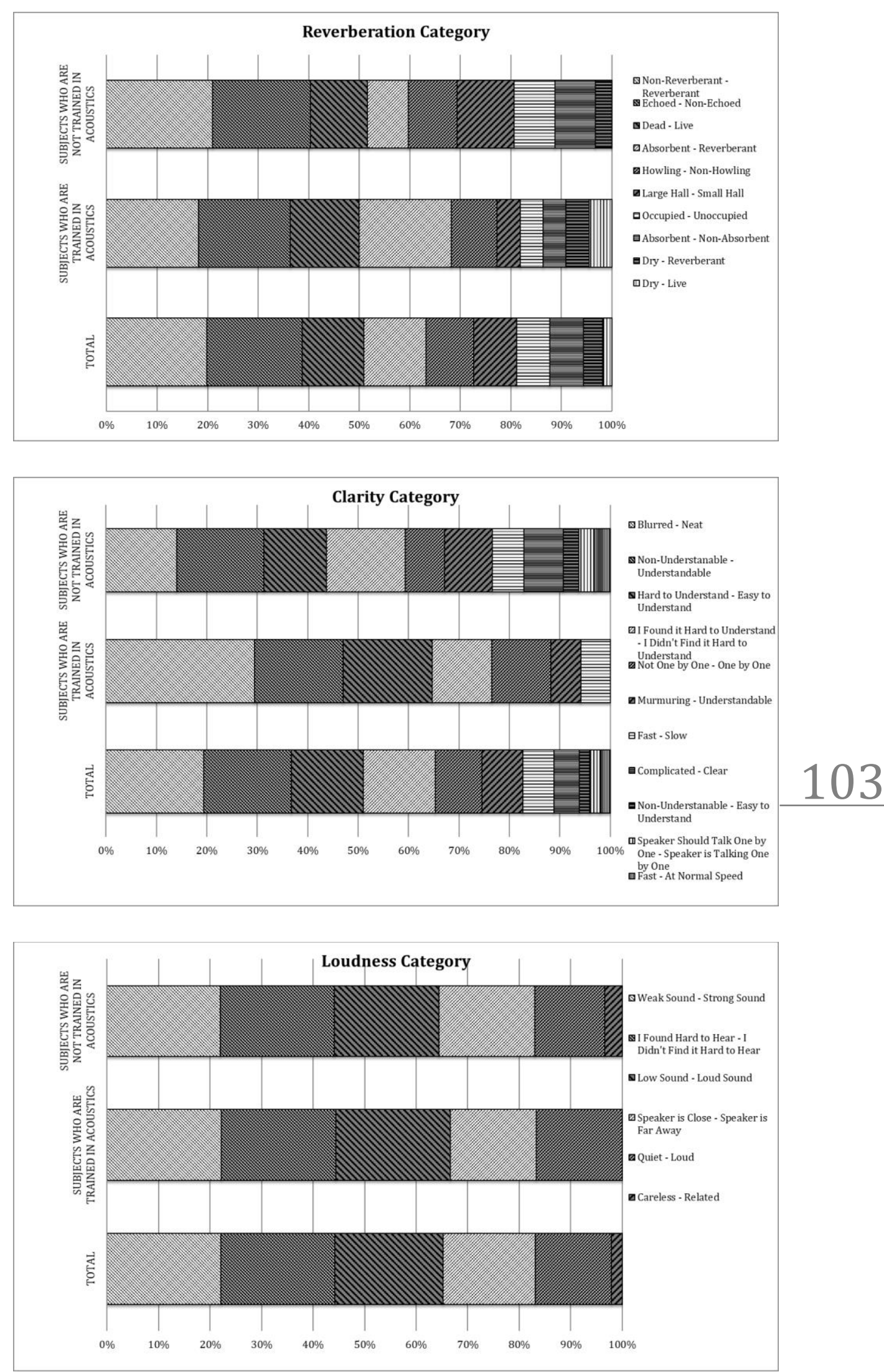


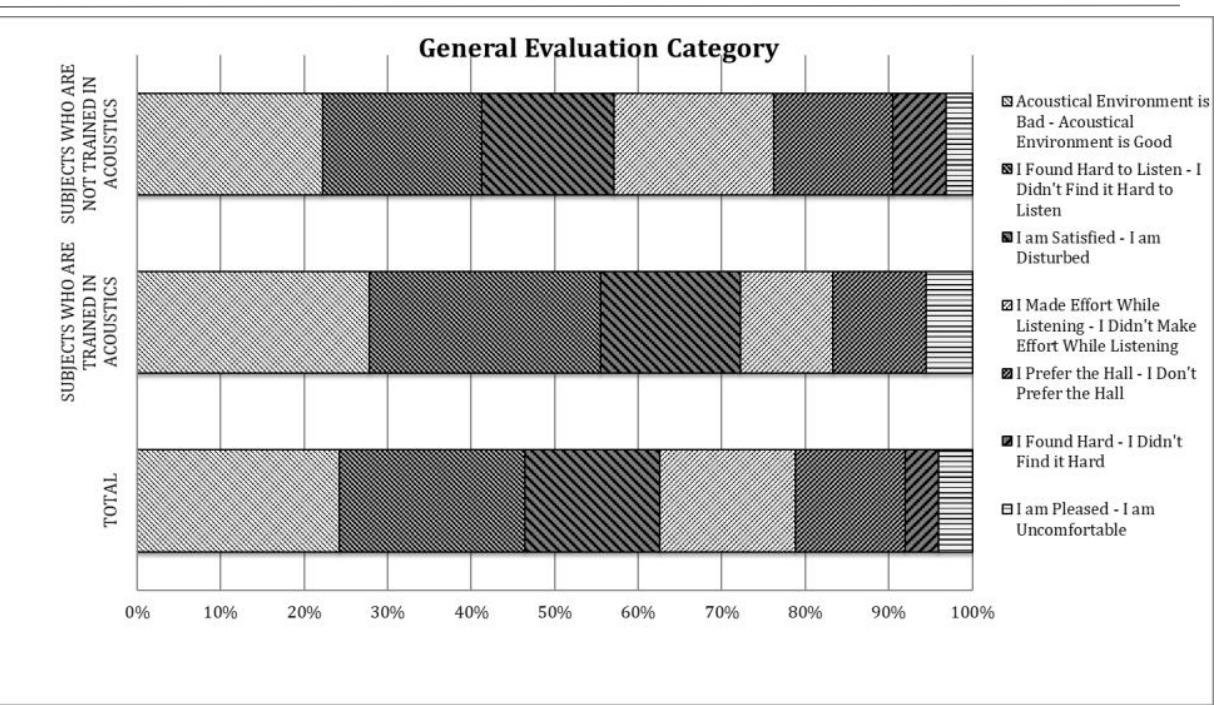

\section{SELECTED ADJECTIVE PAIRS}

In the light of the data obtained from the second step tests, the adjective pair selections made by the subjects are examined.

For the reverberation category, the subjects found the adjective "dry (kuru in Turkish)" to be unsuitable despite the same word is used in subjective evaluations in English often. On the other hand, the subjects considered the adjectives "non-reverberant (çınlamasız in Turkish)" and "dead (ölü in Turkish") appropriate for short reverberation time. However, instead of the adjective pair "complicated - clear (karışık açık in Turkish)" which is obtained from the literature research for the clarity category, the subjects found the "blurred - neat (bulanık - net in Turkish)" adjective pair more appropriate.

Our results show that the subjects are more likely to define the speech sound and the place where the speech is made rather than identifying the speaker. It is also found that those who are not trained in acoustics are more likely to identify more adjective pairs than those who are trained in acoustics, subjects who are trained in acoustics generally made selections less than five for the categories except the general evaluation.

In the second step tests, some highly selected pairs of adjectives contained the same adjectives. This may cause confusion in the questionnaires. It is appropriate to include one of the adjective pairs containing repetitive adjectives in the questionnaires to be prepared.

Therefore, the following adjective pairs are excluded from the list accordingly: a) "absorbent - reverberant (yutucu - çınlamalı in Turkish)" pair is removed from the reverberation category in favor of the "non-reverberant - reverberant (çınlamalı - çınlamasız in Turkish)" pair, b) "hard to understand - easy to understand (anlaşılması zor kolay anlaşılır in Turkish)" pair is removed from the clarity category in favor of the "not-understandable - understandable (anlaşlır anlaşılmaz in Turkish)" pair, c) "murmuring - understandable (mırıltılı anlaşlır in Turkish)" pair is removed from the clarity category in favor
Figure 4. Adjective Pair Selections for General Evaluation Category 
of the "not-understandable - understandable (anlaşılır - anlaşılmaz in Turkish) pair, d) "quiet- loud (sessiz - yüksek sesli in Turkish)" pair is removed from the loudness category in favor of the "low sound - loud sound (alçak ses - yüksek ses in Turkish)" pair.

The resulting adjective pair list is given in Table 7.

Table 7. Adjective pair list (edited)

\begin{tabular}{|c|c|c|}
\hline Category & $\begin{array}{l}\text { Adjective Pair } \\
\end{array}$ & Preference Ratio (\%) \\
\hline \multirow{5}{*}{ 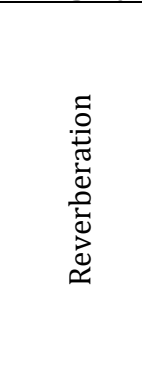 } & $\begin{array}{l}\text { Non-Reverberant - Reverberant } \\
\text { (Çınlamasız - Çınlamalı) }\end{array}$ & 84 \\
\hline & $\begin{array}{l}\text { Echoed - Non-Echoed } \\
\text { (Yankı Var (Yankılı) - Yankı Yok (Yankısız)) }\end{array}$ & 80 \\
\hline & $\begin{array}{l}\text { Dead - Live } \\
\text { (Ölü - Canlı) }\end{array}$ & 52 \\
\hline & $\begin{array}{l}\text { Howling - Non-Howling } \\
\text { Uğultulu - Uğultusuz }\end{array}$ & 40 \\
\hline & $\begin{array}{l}\text { Large Hall - Small Hall } \\
\text { (Büyük Salon - Küçük Salon) }\end{array}$ & 36 \\
\hline \multirow{5}{*}{ 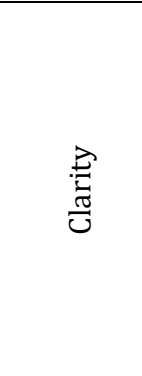 } & $\begin{array}{l}\text { Blurred - Neat } \\
\text { (Bulanık - Net) }\end{array}$ & 76 \\
\hline & $\begin{array}{l}\text { Non-Understandable - Understandable } \\
\text { (Anlașılmaz - Anlașılır) }\end{array}$ & 68 \\
\hline & $\begin{array}{l}\text { Not One by One - One by One } \\
\text { (Tane Tane Değil - Tane Tane) }\end{array}$ & 36 \\
\hline & $\begin{array}{l}\text { Fast - Slow } \\
(\text { Hılı - Yavaș) }\end{array}$ & 24 \\
\hline & $\begin{array}{l}\text { Complicated - Clear } \\
\text { (Karıșik - Açık) }\end{array}$ & 20 \\
\hline \multirow{5}{*}{ 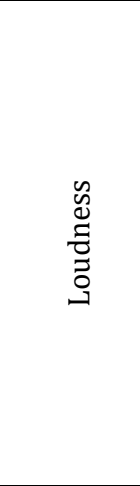 } & $\begin{array}{l}\text { Weak Sound - Strong Sound } \\
\text { (Zayıf Ses - Güçlü Ses) }\end{array}$ & 84 \\
\hline & $\begin{array}{l}\text { I Found it Hard to Hear- I Didn't Find it Hard to } \\
\text { Hear } \\
\text { (Duymakta Zorlandım } \quad-\quad \text { Duymakta } \\
\text { Zorlanmadım) }\end{array}$ & 84 \\
\hline & $\begin{array}{l}\text { Low Sound - Loud Sound } \\
\text { (Alçak Ses - Yüksek Ses) }\end{array}$ & 80 \\
\hline & $\begin{array}{l}\text { Speaker is Close - Speaker is Far Away } \\
\text { (Konuşmacı Yakın Konumda - Konuşmacı Uzak } \\
\text { Konumda) }\end{array}$ & 68 \\
\hline & $\begin{array}{l}\text { Careless - Related } \\
\text { (Umursamaz - İlgili) }\end{array}$ & 8 \\
\hline \multirow{5}{*}{ 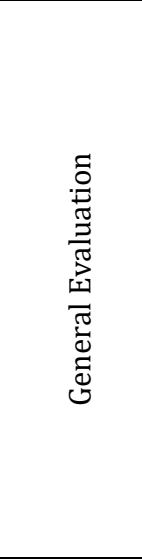 } & $\begin{array}{l}\text { Acoustical Environment is Bad - Acoustical } \\
\text { Environment is Good } \\
\text { (Akustik Ortam Kötü - Akustik Ortam İyi) }\end{array}$ & 96 \\
\hline & $\begin{array}{l}\text { I Found it Hard to Listen - I Didn't Find it Hard } \\
\text { to Listen } \\
\text { (Dinlerken Zorlandım } \quad-\quad \text { Dinlerken } \\
\text { Zorlanmadım) }\end{array}$ & 88 \\
\hline & $\begin{array}{l}\text { I am Satisfied - I am Disturbed } \\
\text { (Memnun Oldum - Rahatsız Oldum) }\end{array}$ & 64 \\
\hline & $\begin{array}{l}\text { I Made Effort While Listening - I Didn't Make } \\
\text { Effort While Listening } \\
\text { (Dinlerken Çaba Gösterdim - Dinlerken Çaba } \\
\text { Göstermedim) }\end{array}$ & 64 \\
\hline & $\begin{array}{l}\text { I Prefer the Hall - I Don't Prefer the Hall } \\
\text { (Salonu Tercih Ederim - Salonu Tercih Etmem) }\end{array}$ & 52 \\
\hline
\end{tabular}

As seen from the resulting table (Table 7), some of the adjective pairs are preferred with a very low percentage despite being in the top five selections. Therefore, we further considered an approach where we 
apply a cut-off limit of $>50 \%$ (Table 8 ). When the cut-off is applied, the number of adjective pairs ended up being less than five for the categories (except the general evaluation) in the questionnaires to be prepared. When the same concepts (scales for categories) are defined with different words, the correlation between these adjective pairs is expected to be high. Therefore, it has been found appropriate to reduce the number of adjective pairs in the questionnaires.

Table 8. List of adjective pairs (cut-off applied)

\begin{tabular}{|c|c|c|}
\hline Category & Adjective Pair & $\begin{array}{l}\text { Preference } \\
\text { Ratio \% }\end{array}$ \\
\hline \multirow{3}{*}{ 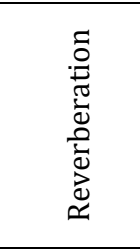 } & $\begin{array}{l}\text { Non-Reverberant - Reverberant } \\
\text { (Çınlamasız - Çınlamalı) }\end{array}$ & 84 \\
\hline & $\begin{array}{l}\text { Echoed - Non-Echoed } \\
\text { (Yankı Var (Yankılı) - Yankı Yok (Yankısız)) }\end{array}$ & 80 \\
\hline & $\begin{array}{l}\text { Dead - Live } \\
\text { (Ölü - Canlı) }\end{array}$ & 52 \\
\hline \multirow{2}{*}{ 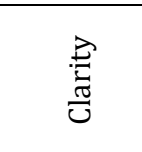 } & $\begin{array}{l}\text { Blurred - Neat } \\
\text { (Bulanık - Net) }\end{array}$ & 76 \\
\hline & $\begin{array}{l}\text { Non-Understandable - Understandable } \\
\text { (Anlașılmaz - Anlașılır) }\end{array}$ & 68 \\
\hline \multirow{4}{*}{ 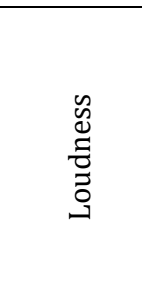 } & $\begin{array}{l}\text { Weak Sound - Strong Sound } \\
\text { (Zayıf Ses - Güçlü Ses) }\end{array}$ & 84 \\
\hline & $\begin{array}{l}\text { I Found it Hard to Hear- I Didn't Find it Hard to Hear } \\
\text { (Duymakta Zorlandım - Duymakta Zorlanmadım) }\end{array}$ & 84 \\
\hline & $\begin{array}{l}\text { Low Sound - Loud Sound } \\
\text { (Alçak Ses - Yüksek Ses) }\end{array}$ & 80 \\
\hline & $\begin{array}{l}\text { Speaker is Close - Speaker is Far Away } \\
\text { (Konuşmacı Yakın Konumda - Konuşmacı Uzak Konumda) }\end{array}$ & 68 \\
\hline \multirow{5}{*}{ 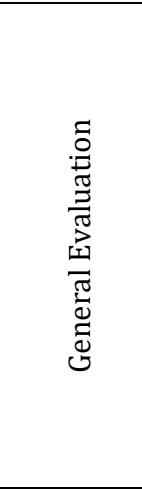 } & $\begin{array}{l}\text { Acoustical Environment is Bad - Acoustical Environment } \\
\text { is Good } \\
\text { (Akustik Ortam Kötü - Akustik Ortam İyi) }\end{array}$ & 96 \\
\hline & $\begin{array}{l}\text { I Found it Hard to Listen - I Didn't Find it Hard to Listen } \\
\text { (Dinlerken Zorlandım - Dinlerken Zorlanmadım) }\end{array}$ & 88 \\
\hline & $\begin{array}{l}\text { I am Satisfied - I am Disturbed } \\
\text { (Memnun Oldum - Rahatsız Oldum) }\end{array}$ & 64 \\
\hline & $\begin{array}{l}\text { I Made Effort While Listening - I Didn't Make Effort While } \\
\text { Listening } \\
\text { (Dinlerken Çaba Gösterdim - Dinlerken Çaba } \\
\text { Göstermedim) }\end{array}$ & 64 \\
\hline & $\begin{array}{l}\text { I Prefer the Hall - I Don't Prefer the Hall } \\
\text { (Salonu Tercih Ederim - Salonu Tercih Etmem) }\end{array}$ & 52 \\
\hline
\end{tabular}

We recommend this final list of descriptive adjective pairs to be included in the questionnaire texts for the subjective evaluations of speech function-halls.

\section{CONCLUSION}

It is important to use a common terminology between the audience and the researcher and / or designer during the subjective evaluation studies within the scope of the acoustic design of the halls. With the hypothesis that translations from different foreign sources do not have the same descriptive effect in one's native language, a two-step study was performed to remedy lack of modifiers for speech sound in Turkish. 
The adjectives and adjective pairs defining speech sound were determined through the choices made by the subjects. Revisions are made to this list according to the interviews with the subjects who have and have not received acoustical training during the study. In the end, a common terminology is established between the listener and the researcher and / or designer and the connection for the flow of information is achieved. With result from the study, it is now possible to present coherent questionnaire texts to the audience for subjective evaluations of speech-function halls during the design or renewal stages of the speech-function halls.

Two step approach designed proved to be effective in terms of communicating with the subjects and obtaining outcome-oriented responses. Indeed, the final recommended list have modifiers came from incorporated subject feedback.

Our survey design revealed the difference between trained versus untrained subjects. We found the recommendations from the trained subjects to be more practical for our purposes in this study. However, giving a concrete recommendation for future studies in terms of using or not using training exercise in their survey design is beyond the scope of this study. It is believed a more comprehensive study with a controlled design of usefulness of questionnaires prepared by trained and untrained subjects would benefit this area of research in subjective evaluations of acoustics parameters.

Architectural acoustics is an inevitable design parameter in order to reach high standards in terms of the success of architectural design and performances based on listening. The results of the study can be used in the field of research to correlate the subjective evaluations with objective quantities obtained from simulations or measurements. Selected modifiers will bridge the gap in between these parameters for speech-function halls.

It is expected this study will contribute to the increase the effectiveness of the activities in the speech-function halls, including the educational spaces and therefore the quality of life in conclusion. This indicates an important social gain. In practice, the study will provide economic benefits by contributing to the design of the halls that do not need improvement afterwards.

This study filled an important gap in this area and will help increase the acoustic comfort conditions in the new and / or existing buildings while reducing financial losses with better service to the customers and with more targeted investments for the owners.

\section{ACKNOWLEDGEMENTS/NOTES}

This article was prepared within the scope of Ezgi Türk Gürkan's ongoing Doctoral Thesis supervised by Zerhan Yüksel Can at Yıldız Technical University. 
Gratitudes to Yıldız Technical University Department of Statistics faculty member Dr. Ömer Bilen, M.Sc Architect Ergin Gürkan and Pro-Plan Proje Mühendislik San. ve Tic. Ltd. Şti. for their support and contributions.

\section{CONFLICT OF INTEREST}

No conflict of interest was declared by the authors.

\section{FINANCIAL DISCLOSURE}

The authors declared that this study has received no financial support.

\section{ETHICS COMMITTEE APPROVAL}

Ethics committee approval was not required for this article.

\section{LEGAL PUBLIC/PRIVATE PERMISSIONS}

In this research, the necessary permissions were obtained from the relevant participants (individuals, institutions and organizations) during the survey, in-depth interview, focus group interview, observation or experiment.

\section{REFERENCES}

Barron, M. (2009). Auditorium Acoustics and Architectural Design. Taylor \& Francis. https://books.google.com.tr/books?id=jPuMAgAAQBAJ

Berber Üçkaya, N. (2014). Eğitim Mekanlarının Akustik Konfor Koşulları Bakımından Írdelenmesi: DEÜ Mimarlık Fakültesi Örneği [Master's Thesis, Dokuz Eylül University].

British Standards Institution. (2009). Acoustics, measurement of room acoustic parameters. Part 1: Performance spaces (ISO 3382-1:2009). BSI Standards, 2009. https://www.iso.org/standard/40979.html

Çoktaş, K. (2019). İşitsel Verilere Dayanarak Mekansal Atmosferin Taşkışla Örneği Üzerinden İncelenmesi [Master's Thesis, Istanbul Technical University].

Farina, A. (2001). Acoustic quality of theatres: Correlations between experimental measures and subjective evaluations. Applied Acoustics, 62(8), 889-916. https://doi.org/10.1016/S0003-682X(00)00082-7

Fields, J. M., Jong, R. G., Flindell, I. H., Gjestland, T., Job, raymond S., Kurra, S., Schuemer-Kohrs, A., Lercher, P., Vallet, M., \& Yano, T. (1998). Recommendation for shared annoyance questions in noise annoyance surveys. Noise Effects '98, 2, 481-486.

Furrer, W., Robinson, E. R., \& Lord, P. (1964). Room and building acoustics and noise abatement. Butterworth. https://books.google.com.tr/books?id=JEDNzQEACAAJ

Gade, A. (2007). Acoustics in Halls for Speech and Music. In T. D. Rossing (Ed.), Springer Handbook of Acoustics (pp. 301-350). Springer New York. https://doi.org/10.1007/978-0-387-30425-0_9 
Harvie-Clark, J., Wallace, D., Dobinson, N., \& Larrieu, F. (2014). Reverberation time, strength \& clarity in school halls: Measurements and modelling. Proceedings of the Institute of Acoustics, 36(3), 121-138.

Kurra, S. (2009). Çevre gürültüsü ve yönetimi. Bahçeșehir Üniversitesi Yayınları.

Kuttruff, H. (1979). Room Acoustics. Applied Science Publishers. https://books.google.com.tr/books?id=dKNsyVAgAYIC

Odeon A / S (2019). Anechoic Recordings. https://odeon.dk/downloads/anechoic-recordings/

Orhan, C. (2019). A Comparative Study on Indoor Soundscape in Museum Environments [Master's Thesis, İhsan Doğramacı Bilkent University].

Özçevik, A. (2012). 'Íşitsel Peyzaj - Soundscape' Kavramı ile Kentsel Akustik Konforun İrdelenmesinde Yeni Bir Yaklaşım [Doctoral Thesis, Yıldız Technical University].

Savcl Özgüven, İ. Z. (2015). İlköğretim Binalarında Konuşma Anlaşılabilirliği ve Ses Kalitesini İncelemek Üzerine Bir Alan Araştırması [Master's Thesis, Istanbul Technical University]. http://hdl.handle.net/11527/8171

Vural, A. (2009). İstanbul'da Bulunan Dört Konser Salonunun Akustik Açıdan Değerlendirilmesi [Master's Thesis, Istanbul Technical University]. http://hdl.handle.net/11527/8199

Yılmaz Karaman, Ö., \& Berber Üçkaya, N. (2015). Eğitim Mekanlarında Akustik Konfor: Dokuz Eylül Üniversitesi Mimarlık Fakültesi Örneği. MEGARON, 10(4), 503-521. https://doi.org/10.5505/megaron.2015.58076

\section{Resume}

Ezgi Türk Gürkan received her B.S Degree in the major of architecture and Master's Degree in Building Physics from Ylldiz Technical University. She currently works as an acoustical consultant in a private firm and is a PhD student in Architecture Faculty at Ylldiz Technical University.

Zerhan Yüksel Can graduated from Istanbul State Engineering and Architectural Academy in 1979 with a B.S. Degree in Architecture and received her Master's Degree in Building Physics from the same university in 1982. She received her Ph.D. from Ylldiz Technical University in 1988, her Associate Professor Degree in 1991 and her Professor Degree in 2001. She is expert in architectural acoustics and soundscape. She worked at Ylldiz Technical University until her retirement in 2018. 